\title{
The Galactic X-ray Halo
}

\author{
J. Pietz ${ }^{1}$, J. Kerp ${ }^{1}$, P.M.W. Kalberla ${ }^{1}$, W.B. Burton ${ }^{2}$, Dap Hartmann ${ }^{2,3}$, \\ and U. Mebold ${ }^{1}$ \\ I Radioastronomisches Institut der Universität Bonn, Germany, \\ 2 Sterrewacht Leiden, The Netherlands \\ 3 Harvard-Smithsonian Center for Astrophysics, USA
}

\begin{abstract}
We analyzed the soft X-ray background (SXRB) source intensity distribution of the $3 / 4 \mathrm{keV}$ and $1 / 4 \mathrm{keV}$ photons by correlating the public $R O S A T$ PSPC All-Sky Survey data with the new Leiden/Dwingeloo H I Survey. We found that the $3 / 4 \mathrm{keV}$ and $1 / 4 \mathrm{keV}$ distant background source intensity distribution can be modelled by an isothermal $\left(T=1.5 \cdot 10^{6} \mathrm{~K}\right)$ disk-like (scale length $A_{1}=15 \mathrm{kpc}$ ) $\mathrm{X}$-ray halo in addition to the radiation of the extragalactic X-ray background.
\end{abstract}

\section{Introduction}

Early investigations of the soft X-ray background at photon energies below $E \leq 1 \mathrm{keV}$ suggested the existence of a hot thermal plasma component, absorbed by the neutral galactic interstellar matter (Nousek et al. 1982, Marshall \& Clark 1984). Using the new Leiden/Dwingeloo H I Survey (Hartmann 1994, Hartmann \& Burton 1997) as quantitative tracer for the photoelectric absorption we analyzed the $1 / 4 \mathrm{keV}$ and $3 / 4 \mathrm{keV}$ data of the ROSAT AllSky Survey (Snowden et al. 1995) to construct an X-ray halo model for the distant hot plasma, explaining the observed X-ray background intensities.

\section{Method}

At photon energies above $1 \mathrm{keV}$ the $\mathrm{X}$-ray background is predominantly of extragalactic origin (Hasinger et al. 1993), whereas at lower energies additional soft X-ray emitting components become important (e.g. Chen et al. 1997). In our approach we made use of the different contributions of three individual spectral soft X-ray background (SXRB) components to the different PSPC energy bands (Kerp \& Pietz, this volume):

- 3/4 keV: comprising an extragalactic, approximated by a power-law $E^{-\Gamma}$ with a photon index of $\Gamma \sim 1.4$, plus a distant hot thermal component with a plasma temperature of $T \sim 1.5 \cdot 10^{6} \mathrm{~K}$; both components are absorbed by the galactic interstellar matter.

- 1/4 keV: consists of extragalactic plus distant thermal radiation, with both components being absorbed by the galactic interstellar matter, and 
the emission of an unabsorbed foreground component with a temperature of $T \sim 0.9 \cdot 10^{6} \mathrm{keV}$.

Because the unabsorbed foreground component accounts only for an insignificant fraction of the $3 / 4 \mathrm{keV}$ emission, the observed intensity distribution in the $3 / 4 \mathrm{keV}$ energy band is caused by the combined effects of photoelectric absorption and "real" X-ray source intensity variations across the sky. Using the Leiden/Dwingeloo $\mathrm{H}$ I survey as a main tracer for the absorption by the galactic interstellar medium - neglecting the absorption by $\mathrm{H}_{2}$ and $\mathrm{HII}$ - it is possible to disentangle both components.

\section{Results}

An analysis of the $3 / 4 \mathrm{keV}$ energy band reveals that the photoelectric absorption cannot account for the observed intensity variations in contrast to the $1.5 \mathrm{keV}$ energy band where we found no intensity variations. We found that a model for the distant thermal component has to fulfill the following conditions:

- The modelled halo X-ray count rate decreases smoothly from the galactic center towards the galactic anticenter.

- The halo X-ray count rate decreases smoothly from the galactic plane to the galactic poles.

In the following, we assume that the galactic $\mathrm{X}$-ray halo is caused by an isothermal hot gas which is in hydrostatic equilibrium with the gravitational potential of the Galaxy. The pressure $p(z)=n(z) T(z)$ is assumed to balance the gravitational potential $\Phi(z)$ :

$$
\left.n(R, z)=n_{0} \cdot \frac{\operatorname{sech}^{2}\left(R / A_{1}\right)}{\operatorname{sech}^{2}\left(R_{\odot} / A_{1}\right)} \exp (-120 \cdot \Phi(z) / T)\right)
$$

According to Taylor \& Cordes (1993) the scale length parameter $A_{1}$ defines a density gradient as function of the galactocentric radius, $R$, normalized to $R_{\odot}=8.5 \mathrm{kpc}$. We used the potential $\Phi(z)$ derived by Kuijken \& Gilmore (1989). The normalization $n_{0}$ is determined by the emission measure assuming a collisional ionization equilibrium plasma model (Mewe-Kaastra). The temperature of the distant plasma is constrained by the count-rate ratios between the $1 / 4 \mathrm{keV}$ and $3 / 4 \mathrm{keV}$ energy bands (Pietz et al. 1997), confirming the used plasma temperatures of $T=1.5 \cdot 10^{6} \mathrm{~K}$. Such a model distribution with best-fit parameters of $n_{0} \approx 1.4 \cdot 10^{-3} \mathrm{~cm}^{-3}$ and $A_{1}=15 \mathrm{kpc}$ can explain simultaneously the background intensity variations of the $3 / 4 \mathrm{keV}$ and $1 / 4$ keV energy bands (see Fig. 1).

\section{Comparison with a Galactic Fountain Model}

Bregman (1980) constructed a galactic fountain model to explain the origin of high velocity clouds (HVCs). Note that the halo parameters of the isothermal 

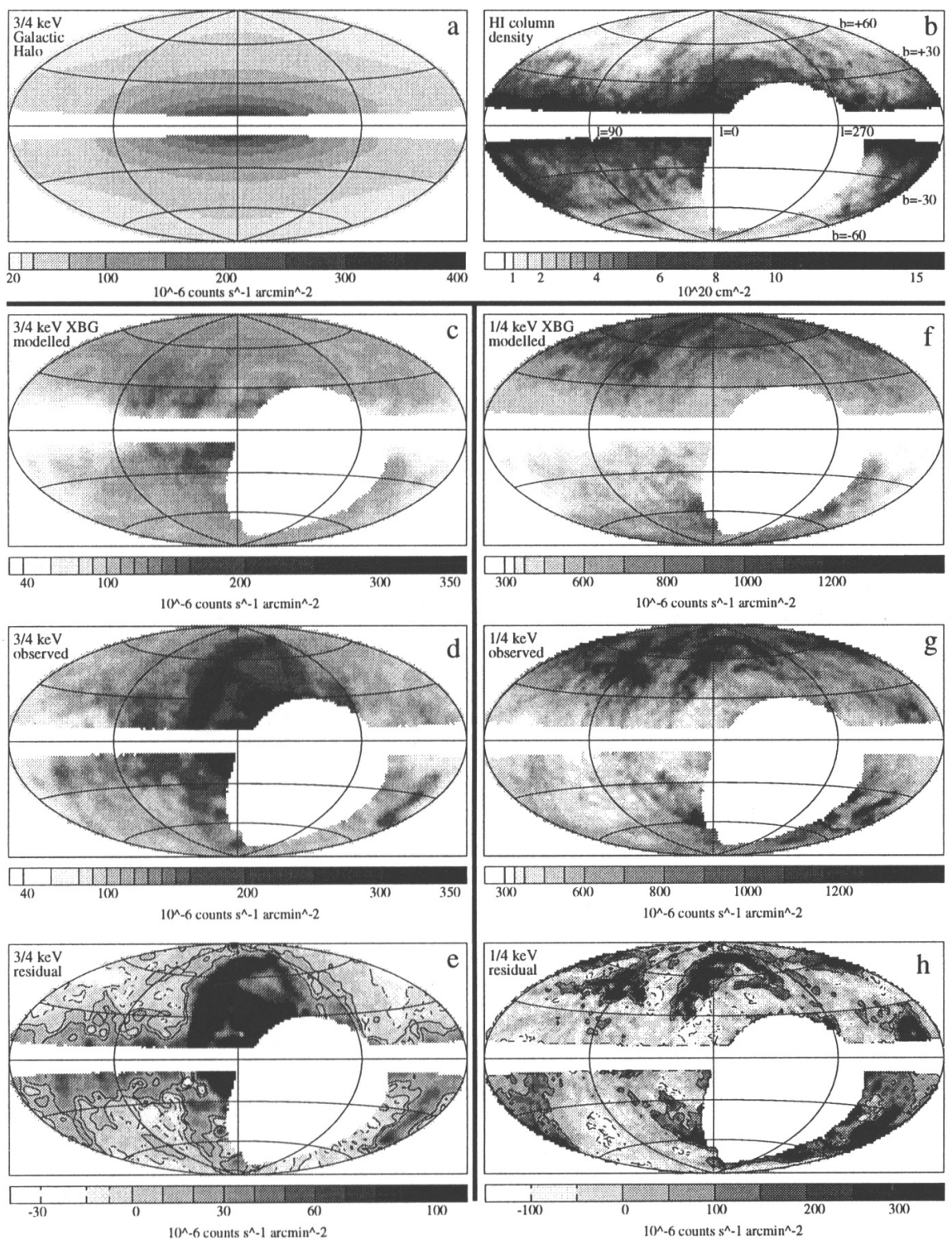

Fig. 1. Comparison of the modelled and observed $3 / 4 \mathrm{keV}$ and $1 / 4 \mathrm{keV}$ SXRB maps centered on the galactic center in Hammer-Aitoff projection. (a) proposed unabsorbed $3 / 4 \mathrm{keV}$ intensity distribution of the distant galactic X-ray halo; (b) observed $\mathrm{H}$ I column density distribution derived from the Leiden/Dwingeloo survey; (c) modelled $3 / 4 \mathrm{keV}$ SXRB intensity distribution; (d) observed $3 / 4 \mathrm{keV}$ intensity distribution; (e) difference map representing observed minus modelled SXRB intensities; (f) modelled SXRB intensity distribution of the $1 / 4 \mathrm{keV}$ energy range; (g) observed $1 / 4 \mathrm{keV}$ intensity distribution; $(\mathrm{h})$ the difference map between the observed X-ray intensity distribution and the model situation. 
model (temperature $T \sim 1.5 \cdot 10^{6} \mathrm{~K}$ and density $n_{0}=1.4 \cdot 10^{-3} \mathrm{~cm}^{-3}$ ) are in the range of the values preferred by Bregman (1980), although his parameters are only valid at the base of the galactic fountain flow, since the temperature decreases with increasing $z$ height in a fountain model. In Bregman's model (1980) radial pressure gradients cause a radial outflow of the rising hot gas, resulting in a center/anticenter asymmetry of the X-ray intensity distribution. Comparison between the count-rate ratios of the isothermal model (Eq. (1)) and the Bregman's galactic fountain model demonstrates that both models can reproduce the observed center/anticenter asymmetry:

\begin{tabular}{c|c|c}
\hline Count-rate ratios & isothermal model & galactic fountain \\
\hline$C\left(l=0^{\circ}, b=30^{\circ}\right)$ & 2.1 & 1.9 \\
\hline$C\left(l=180^{\circ}, b=30^{\circ}\right)$ & $1\left(l=0^{\circ}, b=60^{\circ}\right)$ \\
$C\left(l=180^{\circ}, b=60^{\circ}\right)$ & 1.2 & 1.4 \\
\hline
\end{tabular}

Finally, we analyzed the X-ray shadow of a high-velocity cloud in a similar way as the X-ray shadow analysis of the extragalactic background by galaxies (e.g. Barber et al. 1996). H I spectra of HVC87+41-120, belonging to HVC complex $\mathrm{C}$, were studied to derive the $\mathrm{H}$ I column densities of the high velocity and the low velocity matter. Our result is the detection of an extremely weak HVC X-ray shadow in the PSPC pointing 200721p which suggests that this $\mathrm{HVC}$ is located close to the upper boundary of the galactic halo $(|z|>2.5$ $\mathrm{kpc}$ ) or outside ${ }^{1}$. To estimate the HVC distance by this X-ray shadow we have to know the exact extragalactic $1 / 4 \mathrm{keV}$ count rate, which is still not available with the needed accuracy (e.g. Barber et al. 1996).

\section{References}

Almaini O., et al. (1996): MNRAS 282, 295

Barber, C.R., Roberts, T.P., Warwick, R.S. (1996): MNRAS 282, 157

Bregman, J.N. (1980): ApJ 236, 577

Chen, L.-W., Fabian, A.C., Gendreau, K.C. (1997): MNRAS 285, 449

Hartmann, D. (1994): Ph.D. thesis, University of Leiden

Hartmann, D. \& Burton, W.B. (1997): Atlas of Galactic Neutral Hydrogen, Cambridge University Press

Hasinger G., et al. (1993): A\&A 275, 1

Kuijken, K. \& Gilmore, G. (1989): MNRAS 239, 605

Marshall, F.J. \& Clark, G.W. (1984): ApJ 287, 633

Nousek, J.A., et al. (1982): ApJ 258, 83

Pietz, J., et al. (1997): A\&A submitted

Taylor, J.H. \& Cordes, J.M. (1993): ApJ 411, 674

${ }^{1}$ Note that if the HVC is located outside the galactic X-ray halo the observed X-ray shadow implies a value of $\Gamma=1.5_{-0.5}^{+0.25}$ for the extragalactic power-law index, consistent with the results by Almaini et al. (1996). 